OPEN ACCESS

Edited by:

Samar S. Ayache,

Hôpitaux Universitaires Henn

Mondor, France

Reviewed by:

Mohammad Taheri,

Shahid Beheshti University of Medical

Sciences, Iran

Omid Mirmosayyeb,

Isfahan University of Medical

Sciences, Iran

*Correspondence:

Bin L

jack511@163.com

Specialty section

This article was submitted to

Multiple Sclerosis and

Neuroimmunology,

a section of the journal

Frontiers in Neurology

Received: 31 January 2021

Accepted: 29 June 2021

Published: 31 August 2021

Citation:

Yan $H$, Guo R, Chen W, Xi X, Wang L, Ma J and Li B (2021) Associations of

IRAK1 Gene Polymorphisms and mRNA Expression With NMOSD Risk in the Northern Chinese Han Population. Front. Neurol. 12:661791.

doi: 10.3389/fneur.2021.661791

\section{Associations of IRAK1 Gene Polymorphisms and mRNA Expression With NMOSD Risk in the Northern Chinese Han Population}

\author{
Hongjing Yan ${ }^{1,2,3}$, Ruoyi Guo ${ }^{1,2}$, Weifeng Chen ${ }^{4}$, Xutao $\mathrm{Xi}^{3}$, Lianchang Wang ${ }^{3}$, \\ Jianxun $\mathrm{Ma}^{1,2}$ and $\mathrm{Bin} \mathrm{Li}^{1,2 *}$
}

${ }^{1}$ Department of Neurology, The Second Hospital of Hebei Medical University, Shijiazhuang, China, ${ }^{2}$ Key Laboratory of Hebei Neurology, Shijiazhuang, China, ${ }^{3}$ Department of Neurology, Handan First Hospital, Handan, China, ${ }^{4}$ Department of Neurosurgery, The Central Hospital of Handan, Handan, China

Objectives: Interleukin (IL)-1 receptor-associated kinase 1 (IRAK1) is a very important immunomodulatory gene for autoimmune diseases located on the $\mathrm{X}$ chromosome. However, there was little study about the correlation of IRAK1 functional single nucleotide polymorphisms with mRNA expression in neuromyelitis optica spectrum disorder (NMOSD) patients. In this study, we aimed to investigate the plausible association of IRAK1 polymorphism, IRAK1 mRNA expression, and NMOSD risk in the northern Chinese Han population.

Methods: Four loci of IRAK1 gene (rs1059702, rs7061789, rs1059703, and rs3027898) were genotyped using multiplex SNaPshot technique in 102 NMOSD patients and 213 healthy subjects. Allele, genotype, and haplotype frequencies were compared. Stratified analyses were conducted by age, sex, AQP4 status, and age of onset. IRAK1 mRNA levels in the peripheral blood mononuclear cells of 30 NMOSD patients (of active phase) and 15 healthy control subjects were detected using qPCR. The correlations between the SNP polymorphisms and mRNA expression levels of genes were tested using non-parametric tests.

Results: The minor allele frequencies (MAF) of these four locis were significantly lower in NMOSD cases than that of the controls. The frequencies of rs1059703G/G genotype, rs1059702A/A genotype, rs3027898 C/C genotype, and rs7061789G/G genotype were higher in the case group than that of the control group. Haplotype analysis revealed that the major haplotype "G-A-C-G" (alleles in the order of SNPs rs1059703, rs1059702, rs3027898, and rs7061789), containing the risk alleles, conferred an adverse effect on NMOSD. The level of IRAK1mRNA was markedly higher in NMOSD when compared to the healthy control groups. The IRAK1mRNA levels of female patients with the major haplotype were significantly higher compared to those with other haplotypes and to the male patients with the same genotype. 
Conclusion: IRAK1 polymorphisms were highly correlated with NMOSD susceptibility. Its haplotype G-A-C-G (rs1059703-rs1059702-rs3027898-rs7061789) confers increasing the risk of NMOSD in female patients. The IRAK1 risk haplotype G-A-C-G upregulated IRAK1 mRNA expression in female NMOSD patients. Our study provides a novel insight into the molecular mechanism of the pathogenesis of NMOSD and reveals that IRAK1 is the potential mechanism-specific druggable target in NMOSD disease.

\section{Keywords: IRAK1, polymorphism, NMOSD, mRNA, TLR}

\section{INTRODUCTION}

Neuromyelitis optica spectrum disorder (NMOSD) is a demyelinating autoimmune disease primarily affecting the spinal cord and optic nerve. Compared to multiple sclerosis, Neuromyelitis optica occurs more frequently in women than in men, by as much as $(1,2): 1$ (3). The primary outcomes are physical and visual disabilities. Since the discovery of anti-AQP4 antibodies (AQP4-IgG) in 2004 (4), it is generally believed that NMOSD is characterized by the AQP4-IgG, which are produced by the differentiation of $\mathrm{B}$ cells to plasma cells $(5,6)$, binding to AQP4 on astrocytes $(4,7)$, and cause direct astrocyte injury by antibody-dependent and complement-mediated (cellular) cytotoxicity. However, the exact molecular mechanisms behind the pathogenesis of NMOSD remain unclear. Some studies have shown that toll-like receptor (TLR) signaling, which has been implicated in various neuroimmune processes, may play a relevant role in the pathogenesis of $\operatorname{NMOSD}(1,2,8)$.

Interleukin (IL)-1 receptor-associated kinase 1(IRAK1), which is a vital serine/threonine-protein kinase and the key signal regulator, plays an indispensable role in scaffolding and phosphorylation in TLR signaling pathways (9-13). Recent studies observed an abnormal expression of IRAK1 in several autoimmune diseases, including systemic lupus erythematosus (SLE) and rheumatoid arthritis (RA) $(14,15)$. Zhou et al. found that the levels of IRAK1 transcript in CD4+T cells increased significantly in patients with SLE and were positively associated with disease activity (14). Another work from Ji et al. indicated that inhibition of IRAK1-NF- $\mathrm{KB}$ signaling activity might attenuate the inflammatory activity of MRL/lpr mice and their bone marrow-derived macrophages (15). A previous in vivo experiment disclosed that iguratimod reduced systemic arthritis score, and decreased IRAK1, IL-6, IL-1 $\beta$, TNF- $\alpha$, and IL-17, but enhanced apoptosis in the synovial tissue of a CIA rat model (16). Collectively, these results suggest that IRAK1 may play a vital role in autoimmune diseases, and further study of IRAK1 is warranted.

The IRAK1 gene is located at human locus Xq28. In women, one of the two $\mathrm{X}$ chromosomes which are from each of the two parents, undergoes random X-inactivation in the early stages of embryonic development. Males carry and express only one ChrX, and X chromosome inactivation (XCI) in females provides dosage compensation between the sexes for X-linked genes $(17,18)$. Several studies have demonstrated that the IRAK1 polymorphism may be related to autoimmune diseases, including SLE, RA, and autoimmune thyroid diseases (19-23). These common loci including rs1059702, rs1059703, rs3027898, and rs7061789 were found to be in strong linkage disequilibrium $(22,24)$. Furthermore, these SNPs may be functional SNPs that are likely to play a significant role in modulating the phenotype in females. For example, studies have demonstrated that rs3027898 may be a functional SNP that can regulate IRAK1 mRNA expression $(25,26)$. Another study showed that rs 1059703 may regulate skewed $\mathrm{X}$ chromosome inactivation which is likely to play a vital role in modulating the causes of augmented cell activation (27).

IRAK1 is a gene central to immune regulation. However, its role is less studied in NMOSD. It would be useful to explore the SNP genotypes in IRAK1 and its relation to mRNA expression analysis, and their association with NMSOD. Our study identifies that IRAK1 polymorphisms are correlated with NMOSD risk in northern Chinese Han populations and contribute to the up-regulated expression level of IRAK1 mRNA in female NMOSD patients.

\section{MATERIALS AND METHODS}

\section{Study Subjects}

We enrolled patients diagnosed with NMOSD at the Department of Neurology in the Second Hospital of Hebei Medical University (102 patients including 86 females and 16 males, 30 acute patients including 25 females and five males) from September 2019 to November 2020. Meanwhile, 213 healthy examinees (166 females and 47 males) were enrolled as a healthy control group at the physical examination center. All subjects were from the northern Han Chinese population. It is worth noting that we only enrolled NMOSD patients who fulfilled the 2015 diagnostic criteria for NMOSD (27). Subjects used for determining IRAK1 mRNA expression were active NMOSD patients who had not used hormone or immunomodulatory agents in the previous 6 months. The following exclusion criteria were prescribed: (a) hypertension, diabetes, cerebrovascular disease, tumors, and other chronic diseases; and (b) concurrent with other autoimmune diseases, such as rheumatoid arthritis, systemic lupus erythematosus, and thyroid diseases. All participants signed an informed consent before the study began. Data collection involved extracting relevant details from the patients into a standard case report form, including age, gender, age at onset, AQP4 status, clinical manifestations and initial symptoms, medication history, and disease history. This study was approved by the Ethics Committee of The Second Hospital of Hebei Medical University. 
TABLE 1 | Primer information.

\begin{tabular}{|c|c|c|}
\hline \multirow[t]{2}{*}{ Genes } & \multicolumn{2}{|c|}{ Primer sequence } \\
\hline & Upstream primer & Downstream primer \\
\hline rs1059702 & CTCCTCCGAGAAGTTGTG & GCAGAAGGGAAGAGACAG \\
\hline rs1059703 & CTGGACACGTAGGAGTTC & TGTACGAGAGGCTAGAGAA \\
\hline rs3027898 & AACATGGACTACATCAGGAA & GGCTCTACTTGTGGACTT \\
\hline rs7061789 & AACATGGACTACATCAGGAA & TGATITCAGAACTGGAGATG \\
\hline IRAK1 & AAGGAGAACGCTGACCTGGAGT & CCGTACACCAGGCAGTAGAAGC \\
\hline$\beta$-actin & CACCCAGCACAATGAAGATCAA & CCAGTIITAAATCCTGAGTCAAGC \\
\hline
\end{tabular}

\section{Isolation of DNA and Genotyping by SNaPshot Technique}

We collected $5 \mathrm{ml}$ of peripheral blood in EDTA anticoagulant tubes from each subject. Next, we extracted genomic DNA using the Blood Genomic DNA Extraction Kit (JieRui, Shanghai, China) according to the manufacturer's protocol, and stored it at $-20^{\circ} \mathrm{C}$ for subsequent experiments. DNA was amplified using PCR MIX (Yisheng Biotech Co., Shanghai, China), with focus on four IRAK1 sites (rs1059703-rs1059702-rs3027898rs7061789).The Snapshot kit (ABI, USA) was used for genotyping (28). Table 1 shows the sequences of primers.

\section{qPCR for IRAK1 mRNA}

Furthermore, we enrolled 30 active NMOSD patients and 15 healthy normal controls for examining IRAK1 mRNA expression. We collected a $5 \mathrm{ml}$ fasting EDTA-anticoagulated venous blood sample from each subject. We then isolated peripheral blood mononuclear cells by density-gradient centrifugation using Ficoll-Hypaque (Hao Yang Biological, Tianjin, China). Next, total RNA was isolated using the Total RNApure reagent (ZhuangMeng Biological, Beijing, China) in accordance with the manufacturer's protocol. Complementary DNA (cDNA) was then synthesized from RNA using Prime Script $^{\mathrm{TM}}$ RT reagent Kit with gDNA Eraser (GeneCopoeia, Guangzhou, China) according to the manufacturer's instructions. Real-time quantitative PCR (qPCR) involved the use of qPCR Mix (GeneCopoeia, Guangzhou, China) and was performed on an ABI7300 machine. PCR amplification conditions were: $95^{\circ} \mathrm{C}$ pre-denaturation for $10 \mathrm{~min}, 95^{\circ} \mathrm{C}$ for $15 \mathrm{~s}, 60^{\circ} \mathrm{C}$ for $5 \mathrm{~min}$, and $75^{\circ} \mathrm{C}$ for $20 \mathrm{~s}$, repeated for 40 cycles. Two compound holes were set for each hole. Relative mRNA expression was normalized to the expression of IRAK1 mRNA, and the 2- $\Delta \Delta \mathrm{Ct}$ method was used to calculate the relative quantification of the gene expression level (28).

\section{Statistics}

Results are presented as number (percentage) for frequency and as mean \pm standard deviation for quantitative variables. We used the unpaired Student's $t$-test to compare the differences in means between two groups, and chi-squared tests for categorical variables. Logistic regression analysis was performed to determine the association of IRAK1 SNPs with the risk factors, while one-way analysis of variance (ANOVA) with Bonferroni's post-hoc test was used to compare the differences
TABLE 2 | Demographics and clinical characteristics of participants for IRAK1 SNP.

\begin{tabular}{lccc}
\hline & NMOSD & HC & P-values \\
& $\boldsymbol{n}=\mathbf{1 0 2}$ & $\boldsymbol{n}=\mathbf{2 1 3}$ & \\
\hline Sex, no. (\%) of females & $84.3 \%$ & $77.9 \%$ & 0.185 \\
Age, y (mean \pm SD) & $45.04 \pm 10.86$ & $44.93 \pm 16.82$ & 0.95 \\
Age at onset, y (mean \pm SD) & $43.63 \pm 16.18$ & NA & NA \\
AQP4-lgG+, no. (\%) of patients & $85.29 \%$ & NA & NA \\
Onset symptoms, no. (\%) of patients & & \\
Optic neuritis & $23.30 \%$ & NA & NA \\
Acute myelitis & $48.54 \%$ & NA & NA \\
Brain attacks & $10.67 \%$ & NA & NA \\
Mix attacks & $17.49 \%$ & NA & NA \\
\hline
\end{tabular}

NMOSD, Neuromyelitis optica spectrum disorder; HC, Healthy control group; SD, standard deviation; NA, not assessable. Brain attacks, including the brainstem and brain attacks.

in means among multiple groups. Moreover, we used Pearson's correlations to analyze bivariate correlations. The online software SHEsis (http://analysis2.bio-x.cn/myAnalysis.php) was used for linkage disequilibrium (LD), Hardy-Weinberg balance test, and haplotype (23). SNPStats (https://www.snpstats.net/start.htm) was used to construct haplotypes and analyze the interactions with related factors. The computed statistical power for this study was $0.88(n=126, \mathrm{P} 0=0.2, \alpha=0.05, \mathrm{OR}=2)$ using PASS 15.0. All other statistical analyses were performed using SPSS version 21.0 (IBM Corp., Armonk, NY, USA). $P<0.05$ was considered to be statistically significant.

\section{RESULTS}

\section{Analysis of Clinical Data}

Tables 2, 3 show the characteristics of study participants. In total, we analyzed 315 study participants for SNP, including 213 healthy controls (female 77.9\%) and 102 NMOSD patients (female $84.3 \%$ ). The mean age of NMOSD patients was $45.04 \pm$ 10.86 years, while that of the control group was $44.34 \pm 16.82$ years. This indicates that the mean age or gender distribution was not significantly different between the case and control groups ( $p$ $=0.95)$. Mean age at onset was $43.63 \pm 16.81$. Results indicated that serum anti-AQP4 antibodies were positively detected in 
$87(85.9 \%)$ patients, optic neuritis in $22(23.3 \%)$, myelitis in $48.54 \%$, and brain attacks and mixed attacks in $34(28.16 \%)$. Furthermore, we collected peripheral blood samples from 30 active NMOSD patients (female 25 cases, male five cases) and 15 healthy controls for IRAK1 mRNA expression analysis. Their mean age was $42.5 \pm 15.507$ and $39.6 \pm 9.553$, respectively, which showed no statistical significance. We positively detected serum AQP4-IgG in $23(76.7 \%)$ patients, optic neuritis in 7 (23.3\%) patients, myelitis in $16(53.3 \%)$ patients, brain attacks in $3(10 \%)$ patients, and mix attacks in $4(13.3 \%)$ patients.

\section{Genotyping and HWE}

In this study, we detected four loci (rs1059702, rs7061789, rs1059703, and rs3027898) on the IRAK1 gene. Given that IRAK1 was located on the $\mathrm{X}$ chromosome, we tested all the SNPs for Hardy-Weinberg equilibrium (HWE) only in female participants. Genotypic distributions for all loci were in HardyWeinberg equilibrium ( $p>0.05$ for all), suggesting that the selected sample population was representative (Table 4).

TABLE 3 | Demographics and clinical characteristics of participants for IRAK1 mRNA.

\begin{tabular}{lccc}
\hline & $\begin{array}{c}\text { NMOSD } \\
\boldsymbol{n}=\mathbf{3 0}\end{array}$ & $\begin{array}{c}\text { HC } \\
\boldsymbol{n}=\mathbf{1 5}\end{array}$ & P-values \\
\hline Sex, no. (\%) of females & $83.3 \%$ & $66.6 \%$ & 0.205 \\
Age, y (mean \pm SD) & $42.5 \pm 15.507$ & $39.6 \pm 9.553$ & 0.444 \\
AQP4-lgG+, no. (\%) of patients & $51.1 \%$ & NA & NA \\
Onset symptoms, no. (\%) of patients & & \\
Optic neuritis & $23.30 \%$ & NA & NA \\
Acute myelitis & $53.3 \%$ & NA & NA \\
Brain attacks & $6.7 \%$ & NA & NA \\
Mix attacks & $8.9 \%$ & NA & NA \\
\hline
\end{tabular}

NMOSD, neuromyelitis optica spectrum disorders; HC, healthy controls; SD stands for standard deviation; NA stands for data not available. Brain involvement includes the involvement of the brain stem, cerebrum, and cerebellum.

\section{Correlation Analysis Between rs1059703, rs7061789, rs3027898, or rs1059702 and the NMOSD Risk in Females Under Different Models}

Because of the small sample size of males (with only 16 males), we did not compare data statistically for SNPs on males. Table 5 shows the genotype and allele frequency of the tested four SNPs in the NMOSD case group and the control group. Take rs 1059702 $\mathrm{A}>\mathrm{G}$ for example, the allele $\mathrm{A}$ was the dominant allele due to higher frequency $(76.8 \%)$ compared to the allele $G(12.8 \%)$. The minor allele frequency (MAF) allele $G$ was significantly lower in cases than in controls $(\mathrm{OR}=0.486 ; 95 \% \mathrm{CI}, 0.290-0.813$; $p=0.006)$. In the inheritance dominant model, patients with the AG+GG genotype had a statistically significantly higher risk of NMOSD than healthy controls with the AA genotype after adjusting for age $(\mathrm{OR}=0.48,95 \% \mathrm{CI}=0.27-0.87, p$ $=0.013)$. Similarly, rs7061789, rs1059703, and rs3027898 also showed significantly different alleles and genotype distributions among female patients with NMOSD and healthy controls.

\section{Linkage Disequilibrium and Haplotype Analysis}

The haplotype analysis showed that rs1059702, rs7061789, rs1059703, and rs3027898 were in high linkage disequilibrium (Figure 1). Haplotype analysis identified two common haplotypes with a frequency higher than 0.03: haplotype1 $=$ GAGC with a frequency of $87.75 \%$ in the NMOSD case group and $76.29 \%$ in the healthy control group, and haplotype $2=$ AGAA with a frequency of $10.29 \%$ in the NMOSD case group and $21.13 \%$ in the healthy control group. There was a statistically significant difference in haplotype distribution between NMOSD cases and healthy controls $(p=0.0061)$ (Table 6).

\section{Stratification Analysis Based on Clinical Features of Female NMOSD Patients}

We conducted statistical analysis to evaluate the significance of IRAK1 for SNPs to the listed clinical characteristics

TABLE 4 | Selected SNPs of IRAK1 in this study.

\begin{tabular}{|c|c|c|c|c|c|c|c|c|c|}
\hline \multirow{2}{*}{$\begin{array}{l}\text { SNPs } \\
\text { rs1059702 }\end{array}$} & \multirow{2}{*}{$\begin{array}{l}\text { Location } \\
\text { X:154018741 (GRCh38.p12) }\end{array}$} & \multirow{2}{*}{$\begin{array}{l}\text { Group }(\boldsymbol{n}) \\
\text { Case }(n=86)\end{array}$} & \multicolumn{3}{|c|}{ Genotype (n) } & \multirow{2}{*}{$\begin{array}{c}\boldsymbol{P}_{\text {HWE }} \\
P=0.56\end{array}$} & \multirow{2}{*}{$\begin{array}{l}\text { MAF } \\
0.17\end{array}$} & \multirow{2}{*}{$\begin{array}{l}\text { Nucleotide } \\
A>G\end{array}$} & \multirow{2}{*}{$\begin{array}{l}\text { Functional region } \\
\text { Exon6 }\end{array}$} \\
\hline & & & $\begin{array}{r}\text { AA } \\
66\end{array}$ & $\begin{array}{r}\text { AG } \\
18\end{array}$ & $\begin{array}{c}\mathrm{GG} \\
2\end{array}$ & & & & \\
\hline & & Control $(n=166)$ & 102 & 51 & 13 & $P=0.07$ & & & \\
\hline \multirow[t]{2}{*}{ rs1059703 } & X:154013378 (GRCh38.p12) & Case $(n=86)$ & $\begin{array}{c}\mathrm{AA} \\
2\end{array}$ & $\begin{array}{r}\text { AG } \\
18\end{array}$ & $\begin{array}{c}\text { GG } \\
66\end{array}$ & $P=0.15$ & 0.25 & $G>A$ & Exon12 \\
\hline & & Control $(n=166)$ & 12 & 52 & 102 & $P=0.56$ & & & \\
\hline \multirow[t]{2}{*}{ rs3027898 } & X:154010439 (GRCh38.p12) & Case $(n=86)$ & $\begin{array}{c}\mathrm{AA} \\
3\end{array}$ & $\begin{array}{r}\mathrm{AC} \\
16\end{array}$ & $\begin{array}{r}\text { CC } \\
67\end{array}$ & $P=0.12$ & 0.25 & $\mathrm{C}>\mathrm{A}$ & Downstream \\
\hline & & Control $(n=166)$ & 10 & 50 & 106 & $P=0.22$ & & & \\
\hline \multirow[t]{2}{*}{ rs7061789 } & X:154015024 (GRCh38.p12) & Case $(n=86)$ & $\begin{array}{c}\mathrm{AA} \\
2\end{array}$ & $\begin{array}{r}\text { AG } \\
17\end{array}$ & $\begin{array}{c}\mathrm{GG} \\
67\end{array}$ & $P=0.47$ & 0.24 & $G>A$ & Intron \\
\hline & & Control $(n=166)$ & 10 & 48 & 108 & $P=0.14$ & & & \\
\hline
\end{tabular}

SNPS, single-nucleotide polymorphisms; $P_{H W E}, p$-value for Hardy-Weinberg equilibrium; MAF, minor allele frequency. 
TABLE 5 | Association analysis between SNPs and the female NMOSD risk under different models.

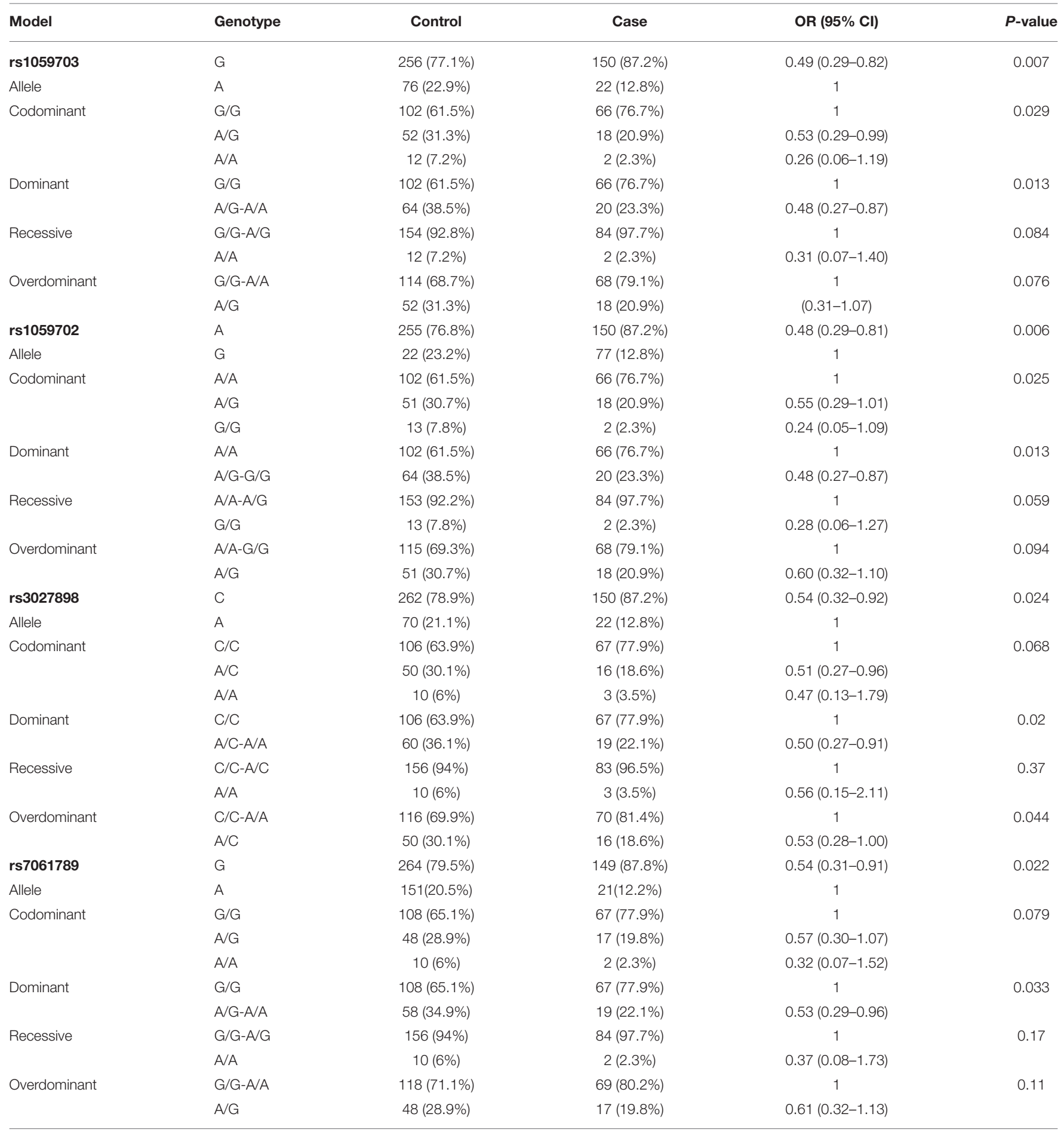

(AQP4 status, onset age, onset symptoms). However, no statistically significant difference was observed in genotype and allele frequencies after stratification by clinical characteristics (Table 7).

\section{Analysis of IRAK1 mRNA Expression}

The relative expression level of IRAK1 mRNA in the NMOSD case group (Figure 2), was statistically significantly higher than in the healthy control group $(p=0.003)$. The level of IRAK1 
mRNA expression was significantly higher in the patents with rs1059703-GG genotype than that with AG genotype ( $p=$ 0.005 , Figure 2). The level of IRAK1 mRNA expression was also significantly higher in female NMOSD patients carrying the risk haplotype GACG (rs1059703-rs1059702-rs3027898-rs7061789) than in those carrying the other haplotypes $(p=0.004)$. In all genotypes, there were increased IRAK1 mRNA expression levels in female NMOSD cases, as compared to males ( $p$ $<0.05, p<0.01$ ). However, no significant differences were observed between male individuals with the GG genotype in the NMOSD case group and in the healthy control group $(p>0.05)$.

\section{DISCUSSION}

The well-established fact that X-linked IRAK1 polymorphisms host susceptibility to autoimmune diseases prompts us to investigate the relevance of IRAK1 gene polymorphism with the risk of NMOSD. In our study, genotype polymorphisms of four common sites in the IRAK1 gene of the northern Chinese Han population were evaluated. The results indicate that both allele and genotype frequencies of the four sites had significant

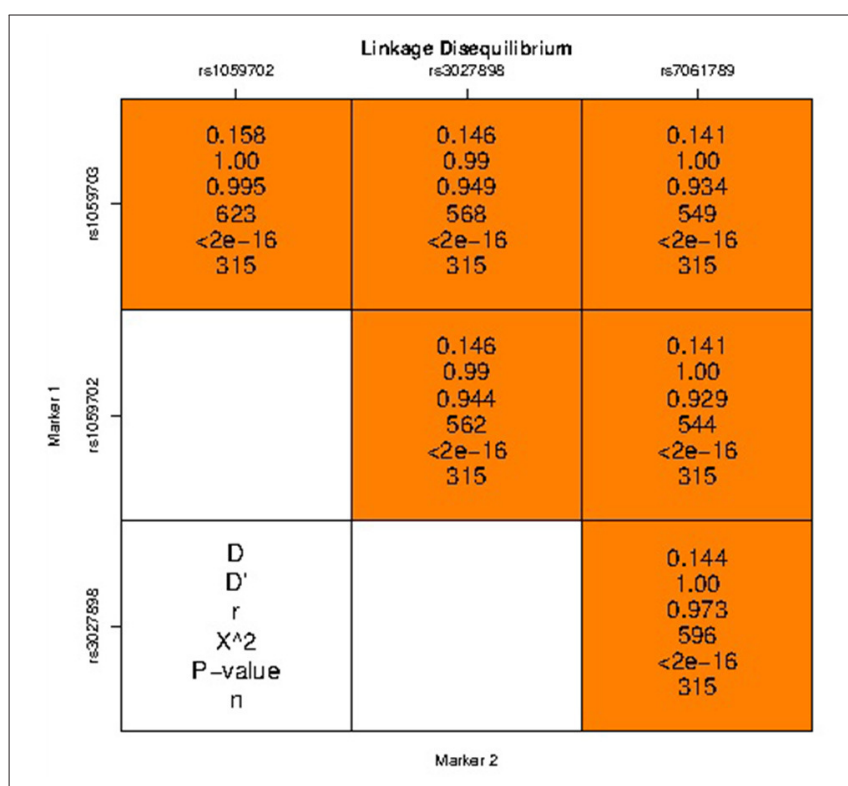

FIGURE 1 | Linkage disequilibrium diagram of four SNP loci in the IRAK1 gene. differences between case and control groups. Also, strong linkage disequilibrium is observed among four polymorphisms. For example, the minor allele frequency $(12.8 \%)$ in the SNP of rs $1059702 \mathrm{~A}>\mathrm{G}$ is lower in NMOSD cases than in controls. Furthermore, the AA genotype of rs1059702 may be a potential risk factor of NMOSD. The lower frequency of the minor allele suggests a protective effect of the minor allele against NMOSD, while the major allele was the risk allele. Subsequent haplotype analysis shows that the haplotypes GACG (rs1059703-rs1059702rs3027898-rs7061789) confers increasing the risk of NMOSD.

Recently, Zhou et al. also found a similar risk association between IRAK1 polymorphisms (rs1059703, rs3027898) and NMOSD (29). The allelic frequencies of rs1059703 and rs3027898 and the linkage disequilibrium relationship between the two SNPs in the control group obtained in our study are quite similar to those described by Zhou et al. (29). Due to a relatively small volume of the case group, the MAF of rs1059703 and rs3027898 was higher in our study compared to those described by Zhou et al., but we achieved a statistical power of $88 \%$. The subjects enrolled in our study were from the northern Han population and those in the study of Zhou et al. were from the Han population from southwest China. Our results agree with those from Zhou. Taken together, the two studies evaluated the association of two common polymorphisms (rs1059703 and rs3027898) in the IRAK1 gene with the risk of NMOSD for Chinese.

Multiple IRAK1 polymorphisms have also been reported to be associated with the susceptibility of several other autoimmune disorders. For example, in a rheumatoid arthritis susceptibility study, the MAF of SNP rs3027898 in the rheumatoid arthritis patient group was significantly higher than in healthy controls and associated with increasing susceptibility to this disease (30). Similarly, the same minor allele has been associated with increasing susceptibility to MS, SLE (23), autoimmune thyroid diseases (29), and rheumatoid arthritis (31). The two polymorphisms, rs1059703 and rs1059702, which are missense mutations and in strong linkage disequilibrium, are associated with autoimmune disease. A previous study showed that rs1059703 T major alleles were increased in RA patients, suggesting $\mathrm{T}$ was a risk factor for $\mathrm{RA}$ in both Tunisian and French women and the rs1059702 C major allele was associated with RA in French women (20). These studies together with the present results indicate that IRAK1 may be a new gene maker of NMOSD. This may be tied to their underlying roles in inflammation disease pathogenesis. IRAK1 possibly plays a key role in the signaling pathways of Toll-like receptors/IL$1 \mathrm{R}$, respectively, activates $\mathrm{NF}-\kappa \mathrm{B}$ signaling pathways and the

TABLE 6 | IRAK1 haplotype association with NMOSD.

\begin{tabular}{|c|c|c|c|c|c|c|c|c|}
\hline & rs1059703 & rs1059702 & rs3027898 & rs7061789 & Control & Case & OR (95\% Cl) & $P$-value \\
\hline 1 & G & A & C & G & 0.7629 & 0.8775 & 1 & - \\
\hline 2 & A & $\mathbf{G}$ & A & A & 0.2113 & 0.1029 & $0.50(0.31-0.81)$ & 0.0049 \\
\hline 3 & A & $G$ & C & G & 0.0188 & 0.0049 & $0.25(0.03-2.05)$ & 0.2 \\
\hline Rare & * & * & * & * & * & * & $2.08(0.48-8.99)$ & 0.33 \\
\hline
\end{tabular}

Global haplotype association p-value: 0.0061, $\left(n=313\right.$, adjusted by sex + age). ${ }^{*}$ represent data is not available. The bold font refers to the statistical significance of result. 
TABLE 7 | Stratified analysis of IRAK1-four SNPs in different clinical features of patients with female NMOSD.

\begin{tabular}{|c|c|c|c|c|c|}
\hline Clinical character & Genotype & Phenotype & Phenotype & OR (95\% Cl) & $P$-value \\
\hline AQP4 status & & AQP4IgG- & AQP4lgG+ & & \\
\hline \multirow[t]{2}{*}{ rs1059703 } & $\mathrm{G} / \mathrm{G}$ & $7(70 \%)$ & $71(79.8 \%)$ & 1 & 0.49 \\
\hline & A/G-A/A & $3(30 \%)$ & 18 (20.2\%) & $0.59(0.14-2.52)$ & \\
\hline \multirow[t]{2}{*}{ rs1059702 } & A/A & $7(70 \%)$ & $71(79.8 \%)$ & 1 & 0.49 \\
\hline & A/G-G/G & $3(30 \%)$ & 18 (20.2\%) & $0.59(0.14-2.52)$ & \\
\hline \multirow[t]{2}{*}{ rs7061789 } & $\mathrm{G} / \mathrm{G}$ & $7(70 \%)$ & 73 (82\%) & 1 & 0.38 \\
\hline & A/G-A/A & $3(30 \%)$ & $16(18 \%)$ & $0.51(0.12-2.19)$ & \\
\hline age of onset & & $\leq 43$ & $>43$ & & \\
\hline \multirow[t]{2}{*}{ rs1059703 } & $\mathrm{G} / \mathrm{G}$ & $43(82.7 \%)$ & 35 (74.5\%) & 1 & 0.32 \\
\hline & $\mathrm{A} / \mathrm{G}-\mathrm{A} / \mathrm{A}$ & 9 (17.3\%) & 12 (25.5\%) & $1.64(0.62-4.33)$ & \\
\hline rs3027898 & A/C-A/A & $9(17.3 \%)$ & $11(23.4 \%)$ & $1.46(0.54-3.91)$ & \\
\hline \multirow[t]{2}{*}{ rs7061789 } & $\mathrm{G} / \mathrm{G}$ & 43 (82.7\%) & 37 (78.7\%) & 1 & 0.62 \\
\hline & A/G-A/A & $9(17.3 \%)$ & $10(21.3 \%)$ & $1.29(0.47-3.52)$ & \\
\hline \multicolumn{6}{|l|}{ Onset symptoms } \\
\hline \multirow[t]{2}{*}{ rs1059703 } & $\mathrm{G} / \mathrm{G}$ & 78 & $1.47(0.13)$ & 0 & 0.61 \\
\hline & A/G-A/A & 21 & $1.62(0.25)$ & $0.14(-0.41-0.70)$ & \\
\hline \multirow[t]{2}{*}{ rs1059702 } & A/A & 78 & $1.47(0.13)$ & 0 & 0.61 \\
\hline & A/G-G/G & 21 & $1.62(0.25)$ & $0.14(-0.41-0.70)$ & \\
\hline \multirow[t]{2}{*}{ rs3027898 } & $\mathrm{C} / \mathrm{C}$ & 79 & $1.49(0.13)$ & 0 & 0.85 \\
\hline & $A / C-A / A$ & 20 & $1.55(0.26)$ & $0.06(-0.51-0.62)$ & \\
\hline rs7061789 & $\mathrm{G} / \mathrm{G}$ & 80 & $1.49(0.13)$ & 0 & 0.76 \\
\hline
\end{tabular}

AQP4lgG+, AQP4lgG positive; AQP4lgG-, AQP4lgG negative; Age at onset Mean $\pm S D$, years $=43.63 \pm 13.32$ years. For stratified according to Onset symptoms set $0=$ optic neuritis, $1=$ myelitis, $2=$ Mix attacks.

release of various inflammatory factors such as IL6, IL-1 $\beta$, and $\mathrm{TNF} \alpha$, which are vital in innate immunity for the molecular and cellular mechanisms of NMOSD. The exact mechanism awaits further investigation.

The tested gene polymorphism loci have been found in the IRAK-1 gene coding and non-coding area, and in one intron area. Although there are few studies of IRAK1 polymorphisms and the association between IRAK1 gene expression in NMOSD, there were several studies showing that rs3027898 was associated with significant downregulation of IRAK1 mRNA levels (25), and the risk genotype of rs1059702 appeared to act to decrease the mRNA levels of MECP2 (included in IRAK1) in SLE patients (26). Rs3027898 was located on 3 ' UTR area of IRAK1. Single-nucleotide polymorphisms located in the 3 'UTR may influence gene expression by varying the interaction between the mRNA and their regulation genes. Thus, to test whether these SNPs associate with mRNA-expression levels, we examined the mRNA expression of IRAK1 and linked SNP haplotypes with the IRAK1 mRNA expression level. The result showed IRAK1 mRNA expression level was affected by rs1059703 genotype, haplotype, and sex. The IRAK1 mRNA expression level was significantly higher in female NMOSD patients carrying the risk haplotype than in those carrying the other haplotypes. This could be due to the effect of the risk haplotype on the interaction between the mRNA and their regulation genes. IRAK1 mRNA expression level was not upregulated in the risk haplotype carrier of males, indicating that IRAK1 polymorphism may regulate mRNA expression by a variety of mechanisms including the skewing of $\mathrm{X}$ chromosome inactivation. Certain $\mathrm{Xic}$ mutations or polymorphisms can result in $\mathrm{X}$ chromosome inactivation skewing, which may result in misexpression between the sexes in diseases. A recent study showed that altered Xchromosome inactivation in $\mathrm{T}$ cells may be associated with sexbiased autoimmune diseases (32). In mammals, $\mathrm{X}$ chromosome inactivation (XCI) seems to be a mechanism to compensate for the dosage difference of X-linked genes between females (XX) and males (XY). It has also been suggested that X-linked genetic polymorphisms together with the skewing of XCI may be the reason for sex-related differences in the phenotype in the female (33). A recent study on trauma showed that the variant IRAK1 haplotype (a risk allele carrier) caused augmented cell activation which may impact cell trafficking during the trauma course (27). Previous animal experiments revealed functional variability in cellular mosaicism for IRAK1 expression and natural X-linked 
A

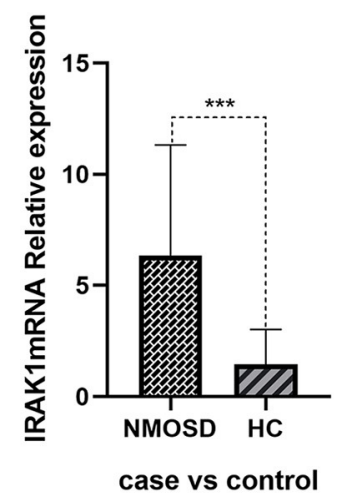

E

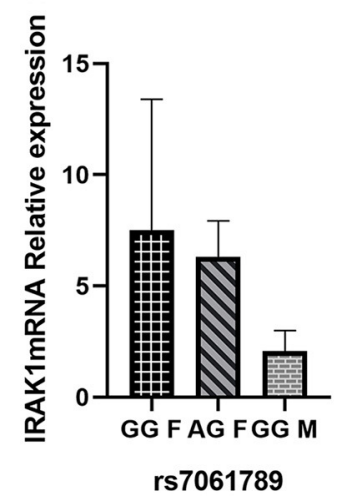

B

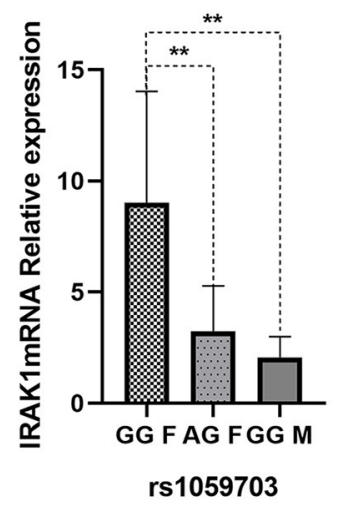

F

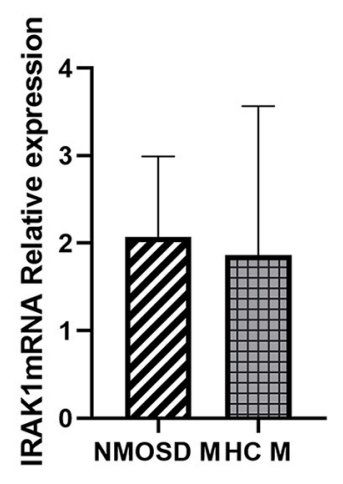

C

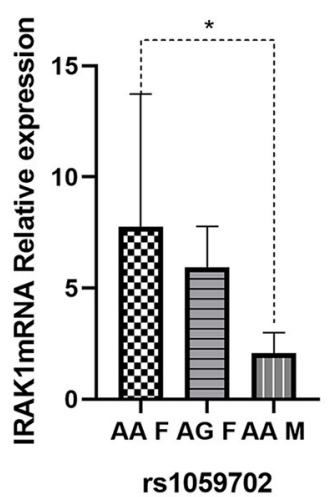

G

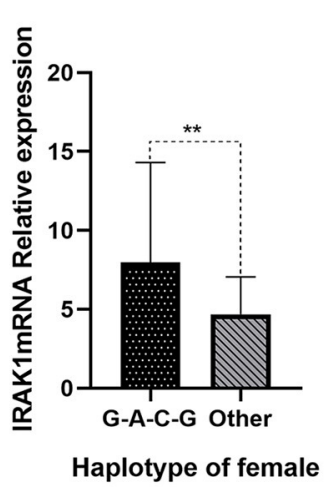

D

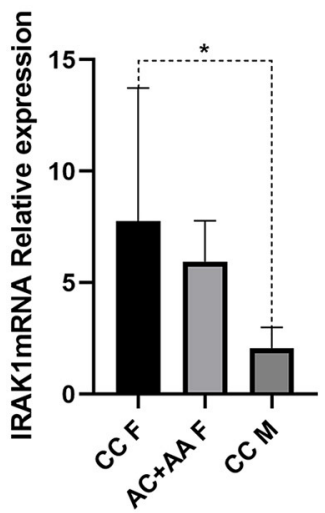

rs3027898

H

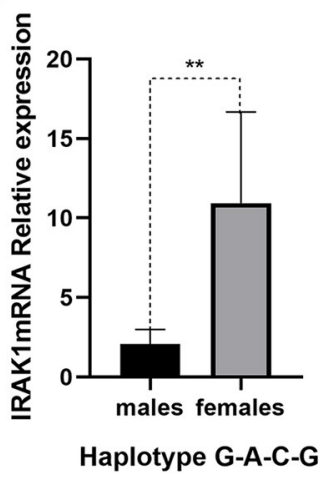

FIGURE 2 | (A) Expression of IRAK1 mRNA level in NMOSD case group and healthy control group. (B) Expression of IRAK1 mRNA level in NMOSD patients with different rs1059703-genotypes. (C) Expression of IRAK1 mRNA level in NMOSD patients with different rs1059702-genotypes. (D) Expression of IRAK1 mRNA level in NMOSD patients with different rs3027898-genotypes. (E) Expression of IRAK1 mRNA level in NMOSD patients with different rs7061789-genotypes. (F) Expression of IRAK1 mRNA level in male NMOSD patients and male healthy control group. (G) Expression of IRAK1 mRNA level in female NMOSD patients with different haplotypes. (H) Expression of IRAK1 mRNA level in male and female NMOSD patients with the risk haplotype. ${ }^{\star} P<0.05,{ }^{\star \star} P<0.01,{ }^{\star \star \star} P<0.001$.

polymorphisms during sepsis. The exact mechanisms of IRAK1 DNA polymorphisms regulating IRAK1 mRNA expression are still not known. Further study focusing on IRAK1 SNP function is highly desirable in the future.

The actual number of NMOSD cases in our sample is somewhat on the low side; in particular, male patients are difficult to recruit because of the low disease incidence in the population. Nevertheless, there is no published report on the association between the IRAK1 haplotype GACG (rs1059703rs1059702-rs3027898-rs7061789) polymorphisms and the mRNA expression of this gene, and our attempt is the first study in this topic. Furthermore, there is currently no evidence that IRAK1 polymorphisms may regulate the inactivation of genes on the $\mathrm{X}$ chromosome. We still need the validation cohort study to further confirm our results.

In conclusion, IRAK1 polymorphisms were highly correlated with NMOSD susceptibility. Its haplotype GACG (rs1059703rs1059702-rs3027898-rs7061789) confers increasing the risk of NMOSD in females. The IRAK1 risk haplotype GACG might upregulate IRAK1 mRNA expression in female NMOSD patients. Our study provides a novel insight into the molecular mechanism of the pathogenesis of NMOSD and reveals that IRAK1 is the potential mechanism-specific druggable target in NMOSD disease.

\section{DATA AVAILABILITY STATEMENT}

The data presented in the study are deposited in the https://www.ebi.ac.uk/eva repository, accession number Project: PRJEB46209, Analyses: ERZ2822693.

\section{ETHICS STATEMENT}

The studies involving human participants were reviewed and approved by the Clinical Research Ethical Committee of the Second Hospital of Hebei Medical University. The 
patients/participants provided their written informed consent to participate in this study.

\section{AUTHOR CONTRIBUTIONS}

BL designed the research, reviewed, revised, and edited the article. BL and HY conducted and coordinated the research. WC and RG performed the analysis and modeling of the data. HY drafted the article. XX and LW contributed to the Materials and Methods section and the figures. All authors contributed to the research and writing of the article.

\section{REFERENCES}

1. Dias ASO, Sacramento PM, Lopes LM, Sales MC, Castro C, Araújo ACRA, et al. TLR-2 and TLR-4 agonists favor expansion of CD4(+) $\mathrm{T}$ cell subsets implicated in the. Mult Scler Relat Dis. (2019) 34:6676. doi: 10.1016/j.msard.2019.06.018

2. Shimizu M, Okuno T, Kinoshita M, Sumi H, Fujimura H, Yamashita K, et al. Mitochondrial DNA enhance innate immune responses in neuromyelitis optica by. Sci Rep. (2020) 10:13274. doi: 10.1038/s41598-020-70203-x

3. Borisow N, Kleiter I, Gahlen A, Fischer K, Wernecke K, Pache F, et al. Influence of female sex and fertile age on neuromyelitis optica spectrum disorders. Mult Scler. (2017) 23:1092-103. doi: 10.1177/1352458516671203

4. Lennon VA, Wingerchuk DM, Kryzer TJ, Pittock SJ, Lucchinetti CF, Fujihara K, et al. A serum autoantibody marker of neuromyelitis optica: distinction from multiple sclerosis. Lancet. (2004) 364:210612. doi: 10.1016/S0140-6736(04)17551-X

5. Cotzomi E, Stathopoulos P, Lee CS, Ritchie AM, Soltys JN, Delmotte FR, et al. Early B cell tolerance defects in neuromyelitis optica favour anti-AQP4 autoantibody. Brain. (2019) 142:1598-615. doi: 10.1093/brain/awz106

6. Wilson R, Makuch M, Kienzler A-K, Varley J, Taylor J, Woodhall M, et al. Condition-dependent generation of aquaporin-4 antibodies from circulating B cells in neuromyelitis optica. Brain. (2018) 141:106374. doi: 10.1093/brain/awy010

7. Lennon VA, Kryzer TJ, Pittock SJ, Verkman AS, Hinson SR. IgG marker of optic-spinal multiple sclerosis binds to the aquaporin-4 water channel. J Exp Med. (2005) 202:473-7. doi: 10.1084/jem.20050304

8. Barros PO, Dias A, Kasahara TM, Ornelas A, Aguiar RS, Leon SA, et al. Expansion of IL-6(+) Th17-like cells expressing TLRs correlates with microbial translocation and neurological disabilities in NMOSD patients. $J$ Neuroimmunol. (2017) 307:82-90. doi: 10.1016/j.jneuroim.2017.04.001

9. Fonte E, Agathangelidis A, Reverberi D, Ntoufa S, Scarfò L, Ranghetti P, et al. Toll-like receptor stimulation in splenic marginal zone lymphoma can modulate cell signaling, activation and proliferation. Haematologica. (2015) 100:1460-8. doi: 10.3324/haematol.2014.119933

10. Chiang EY, Yu X, Grogan JL. Immune complex-mediated cell activation from systemic lupus erythematosus and rheumatoid arthritis patients elaborate different requirements for IRAK1/4 kinase activity across human cell types. J Immunol. (2011) 186:1279-88. doi: 10.4049/jimmunol.1002821

11. Kawagoe T, Sato S, Matsushita K, Kato H, Matsui K, Kumagai Y, et al. Sequential control of Toll-like receptor-dependent responses by IRAK1 and IRAK2. Nat Immunol. (2008) 9:684-91. doi: 10.1038/ni.1606

12. $\mathrm{Su} \mathrm{LC}, \mathrm{Xu} \mathrm{WD}$, Huang AF. IRAK family in inflammatory autoimmune diseases. Autoimmun Rev. (2020) 2020:102461. doi: 10.1016/j.autrev.2020.102461

13. Ghosh S, Dass JFP. Study of pathway cross-talk interactions with NF-кB leading to its activation via ubiquitination or phosphorylation: a brief review. Gene. (2016) 584:97-109. doi: 10.1016/j.gene.2016.03.008

14. Zhou Z, Tian Z, Zhang M, Zhang Y, Ni B, Hao F. Upregulated IL-1 receptor-associated kinase 1 (IRAK1) in systemic lupus erythematosus: IRAK1 inhibition represses Th17 differentiation with therapeutic potential. Immunol Invest. (2018) 47:468-83. doi: 10.1080/08820139.2018. 1458105

\section{FUNDING}

The study was supported by the Hebei Province Science and Technology Project (20200040).

\section{ACKNOWLEDGMENTS}

We would like to extend our deepest appreciation to all of the institutional managers for assisting us in this research and to all of the nurses who were kind enough to participate in this survey.

15. Ji L, Fan X, Hou X, Fu D, Bao J, Zhuang A, et al. Jieduquyuziyin prescription suppresses inflammatory activity of MRL/lpr mice and their bone marrowderived macrophages via inhibiting expression of IRAK1-NF-кB signaling pathway. Front Pharmacol. (2020) 11:1049. doi: 10.3389/fphar.2020.01049

16. Kong R, Gao J, Ji L, Peng Y, Zhang J, Zhao D. Iguratimod ameliorates rheumatoid arthritis progression through regulating miR-146a mediated IRAK1 expression and TRAF6/JNK1 pathway: an in vivo and in vitro study. Clin Exp Rheumatol. (2021) 39:289-303.

17. Spolarics Z. The X-files of inflammation: cellular mosaicism of X-linked polymorphic genes and the female advantage in the host response to injury and infection. Shock. (2007) 27:597-604. doi: 10.1097/SHK.0b013e31802e40bd

18. Belmont JW. Genetic control of $\mathrm{X}$ inactivation and processes leading to X-inactivation skewing. Am J Hum Genet. (1996) 58:1101-8.

19. Zhai $\mathrm{Y}, \mathrm{Xu} \mathrm{K}$, Leng $\mathrm{R}$, Cen $\mathrm{H}$, Wang $\mathrm{W}$, Zhu $\mathrm{Y}$, et al. Association of interleukin-1 receptor-associated kinase (IRAK1) gene polymorphisms (rs3027898, rs1059702) with systemic lupus erythematosus in a Chinese Han population. Inflamm Res. (2013) 62:555-60. doi: 10.1007/s00011-013-0607-2

20. Khalifa O, Balandraud N, Lambert N, Auger I, Roudier J, Sénéchal A, et al. TMEM187-IRAK1 polymorphisms associated with rheumatoid arthritis susceptibility in Tunisian and French female populations: influence of geographic origin. J Immunol Res. (2017) 2017:4915950. doi: 10.1155/2017/4915950

21. Jacob CO, Zhu J, Armstrong DL, Yan M, Han J, Zhou XJ, et al. Identification of IRAK1 as a risk gene with critical role in the pathogenesis of systemic lupus erythematosus. Proc Natl Acad Sci USA. (2009) 106:625661. doi: 10.1073/pnas.0901181106

22. Shin H, Cho WK, Baek I, Lee NY, Lee YJ, Kim SK, et al. Polymorphisms of IRAK1 gene on $\mathrm{X}$ chromosome is associated with hashimoto thyroiditis. Endocrinology. (2020) 161:bqaa088. doi: 10.1210/endocr/bqaa088

23. Song R, Qin Q, Yan N, Muhali F, Meng S, He S, et al. Variants in IRAK1MECP2 region confer susceptibility to autoimmune thyroid diseases. Mol Cell Endocrinol. (2015) 399:244-9. doi: 10.1016/j.mce.2014.10.013

24. Singh S, Rai G, Aggarwal A. Association of microRNA-146a and its target gene IRAK1 polymorphism with enthesitis related arthritis category of juvenile idiopathic arthritis. Rheumatol Int. (2014) 34:1395-400. doi: 10.1007/s00296-014-3001-7

25. Vreca M, Andjelkovic M, Tosic N, Zekovic A, Damjanov N, Pavlovic S, et al. Impact of alterations in X-linked IRAK1gene and miR-146a on susceptibility and clinical manifestations in patients with systemic sclerosis. Immunol Lett. (2018) 204:1-8. doi: 10.1016/j.imlet.2018.10.002

26. Kaufman KM, Zhao J, Kelly JA, Hughes T, Adler A, Sanchez E, et al. Fine mapping of Xq28: both MECP2 and IRAK1 contribute to risk for systemic lupus erythematosus in multiple ancestral groups. Ann Rheum Dis. (2013) 72:437-44. doi: 10.1136/annrheumdis-2012-201851

27. Morcillo P, Qin Y, Peña G, Mosenthal AC, Livingston DH, Spolarics Z Directional X chromosome skewing of white blood cells from subjects with heterozygous mosaicism for the variant IRAK1 Haplotype. Inflammation. (2020) 43:370-81. doi: 10.1007/s10753-019-01127-6

28. Mehta B, Daniel R, Phillips C, McNevin D. Forensically relevant SNaPshot $\left({ }^{\circledR}\right)$ assays for human DNA SNP analysis: a review. Int J Legal Med. (2017) 131:21-37. doi: 10.1007/s00414-016-1490-5 
29. Zhang $\mathrm{H}, \mathrm{Pu}$ J, Wang $\mathrm{X}$, Shen $\mathrm{L}$, Zhao G, Zhuang $\mathrm{C}$, et al. IRAK1 rs3027898 C/A polymorphism is associated with risk of rheumatoid arthritis. Rheumatol Int. (2013) 33:369-75. doi: 10.1007/s00296-01 2-2379-3

30. Labib DA, Shaker OG, El Refai RM, Ghoniem SA, Elmazny A. Association between miRNA-146a and polymorphisms of its target gene, IRAK1, regarding susceptibility to and clinical features of systemic lupus erythematous and multiple sclerosis. Lab Med. (2019) 50:34-41. doi: 10.1093/labmed/lmy033

31. Syrett CM, Paneru B, Sandoval-Heglund D, Wang J, Banerjee S, Sindhava V, et al. Altered X-chromosome inactivation in $\mathrm{T}$ cells may promote sex-biased autoimmune diseases. JCI insight. (2019) 4:e126751. doi: 10.1172/jci.insight.1 26751

32. Cotton AM, Ge B, Light N, Adoue V, Pastinen T, Brown CJ. Analysis of expressed SNPs identifies variable extents of expression from the human. Genome Biol. (2013) 14:R122. doi: 10.1186/gb-2013-1411-r122

33. Chandra R, Federici S, Németh ZH, Csóka B, Thomas JA, Donnelly $\mathrm{R}$, et al. Cellular mosaicism for $\mathrm{X}$-linked polymorphisms and IRAK1 expression presents a distinct phenotype and improves survival following sepsis. J Leukocyte Biol. (2014) 95:497-507. doi: 10.1189/jlb. 0713397

Conflict of Interest: The authors declare that the research was conducted in the absence of any commercial or financial relationships that could be construed as a potential conflict of interest.

Publisher's Note: All claims expressed in this article are solely those of the authors and do not necessarily represent those of their affiliated organizations, or those of the publisher, the editors and the reviewers. Any product that may be evaluated in this article, or claim that may be made by its manufacturer, is not guaranteed or endorsed by the publisher.

Copyright (C) 2021 Yan, Guo, Chen, Xi, Wang, Ma and Li. This is an open-access article distributed under the terms of the Creative Commons Attribution License (CC $B Y)$. The use, distribution or reproduction in other forums is permitted, provided the original author(s) and the copyright owner(s) are credited and that the original publication in this journal is cited, in accordance with accepted academic practice. No use, distribution or reproduction is permitted which does not comply with these terms. 